Abstracted/indexed in Academic Search Complete, Asia Journals Online, Bangladesh Journals Online, Biological Abstracts, BIOSIS Previews, CAB Abstracts, Current Abstracts, Directory of Open Access Journals, EMBASE/Excerpta Medica, Google Scholar, HINARI (WHO), International

\title{
Anti-tumor effect of Huayu Jiedu formula on SGC-7901 cancer cell
}

\author{
Wei Zheng', 2, Zhang Junping², Zhang Aihua², Fan Junming², Wang Yanchun ${ }^{3}$ \\ and Liu Yanqing 4 \\ ${ }^{1}$ Nanjing University of Chinese Medicine, Nanjing, Jiangsu, P. R. China; ${ }^{2}$ Academy Institutes of Traditional Chinese \\ Medicine, Zhengzhou, Henan, P. R. China; ${ }^{3}$ Henan Province People's Hospital, Zhengzhou, Henan, P. R. China; \\ ${ }^{4}$ Yangzhou University, Yangzhou 225009, Jiangsu, P. R. China.
}

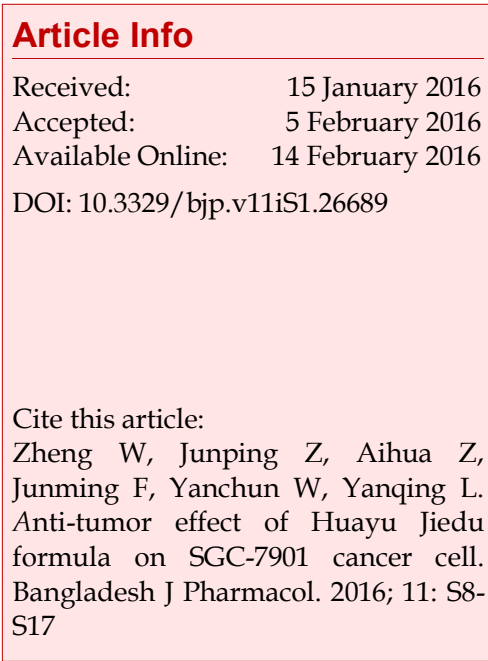

\begin{abstract}
In this study, we evaluated the effects of the extract of Huayu Jiedu (HYJD) formula on anti-tumor activity. It was found that HYJD treatment inhibited proliferation of seven cancer cell lines $\left(\mathrm{IC}_{50}: 1.23-6.23 \mathrm{mg} / \mathrm{mL}\right)$. The gastric cancer cell line SGC-7901 was the most sensitive to HYJD. HYJD inhibited SGC-7901 cells proliferation in a dose- and time-dependant manner. Moreover, HYJD significantly inhibited SGC-7901 cells adhesion, invasion and migration in a dose-dependent manner. qPCR results showed that HYJD treatment had no effect on PI3K, PDK1 and Akt mRNA expression. However, HYJD dramatically down-regulated Akt phosphorylation through inhibition of PDK1 kinase activity. Importantly, HYJD inhibited growth of tumor xenografts with no effect on body weight and spleen index. HYJD also significantly down-regulated Akt phosphorylation in tumor tissue. Taken together, this study demonstrated that HYJD exhibited an anti-tumor property, suggesting that HYJD may be a potential drug in the treatment of cancer.
\end{abstract}

\section{Introduction}

Gastric cancer is the fourth most common malignant tumor and the second leading cause of cancer-related mortality in all kinds of cancers worldwide (Jemal et al, 2011). There is marked geographic variation, with high incidence rates in Eastern Asia, Eastern Europe, and South America, and the lowest rates in North America and Africa (Siegel et al, 2014).

Traditional Chinese Medicine has been applied in many fields of humanity health for thousands of years with its excellent achievements being increasingly acknowledged all over the world ( $\mathrm{Li}$ et al, 2013). Huayu Jiedu

This paper was presented in the 3rd International Conference on Biomedicine and Pharmaceutics in Zhuhai, China, on December 11-13, 2015.
(HYJD) formula has been widely used for treatment of diseases such as hepatitis and rheumatoid arthritis with the action of supplementing the kidney, removing blood stasis and detoxicating (Cao et al, 2008). The recent study reported that HYJD formula in combination with chemotherapy could synergistically inhibit transplanted hepatocarcinoma in mice (Cao et al, 2005). As a TCM formula, HYJD consists of six Chinese medicines with percentages in weight as follows: Sculellaria barbata (50\%), Costus root (16\%), endothelium corneum gigeriae galli $(16 \%)$, Pseudo-ginseng (8\%), Gekko japonicus (5\%), and scorpion (5\%). Our clinical research found that HYJD could obviously improve clinical symptoms, quality of life and prolong the life span of cancer patients. However, the anticancer mechanism is still unknown.

In this study, we found HYJD inhibited SGC-7901 cell 
proliferation, adhesion, invasion, migration and tumor growth through down-regulation of AKT phosphorylation, suggesting that HYJD may be a potential drug in the treatment of gastric cancer.

\section{Materials and Methods \\ Extraction and HPLC analysis}

The extract of HYJD is prepared as follows: In brief, 122 $\mathrm{g}$ of HYJD was smashed as coarse dust and then extracted twice with boiling water of about 8 times as the formula. The two decoctions were mixed, then the mixture was concentrated prior to dry into powder. HYJDE was analyzed by high performance liquid chromatography (HPLC). HPLC analysis was performed on an Angilent 1260 series HPLC apparatus equipped with a DAD detector. A Waters C18 column $(150 \mathrm{~mm} \times 4.6 \mathrm{~mm}$ I.D., $5 \mu \mathrm{m})$ was used for analysis, and the detection wavelength was set at $335 \mathrm{~nm}$. The mobile phase consisted of methanol and water (contains $0.05 \%$ formic acid). A $10 \mu \mathrm{L}$ aliquot of each sample solution was injected and eluted according to the following program, described in terms of the percentage of methanol present, at a flow rate of $1.0 \mathrm{~mL} / \mathrm{min}$ : $25-45 \%$ from 0 to $30 \mathrm{~min}, 45-80 \%$ from 30 to $50 \mathrm{~min}$.

\section{Cell culture and reagents}

SGC-7901, HepG2, MCF-7, ECA-109, Caco-2, Hela and A549 cell lines were obtained from the Type Culture Collection of the Chinese Academy of Sciences and cultured in Dulbecco's modified Eagle's medium (DMEM) and RPMI1640 medium (Thermo Scientific, Waltham, MA) containing $10 \%$ fetal bovine serum (GIBCO, Grand Island, NY), $100 \mathrm{U} / \mathrm{mL}$ penicillin and $100 \mathrm{U} / \mathrm{mL}$ streptomycin (Sigma, USA) in a humidified atmosphere of $5 \% \mathrm{CO}_{2}$ at $37^{\circ} \mathrm{C}$. 3-(4, 5-dimethylthiazol-2 -yl) 2, 5-diphenyltetrazolium bromide (MTT), methyl sulfoxide (DMSO), fibronectin, crystal violet and cyclophosphamide (CTX) were purchased from SigmaAldrich (Saint Louis, MO, USA). Anti-AKT (\#4691), anti -PDK1 (\#5662), anti-PI3K (\#4225), anti-phospho-AKT (\#4058) anti-phospho-PDK1 (\#3061), anti-phospho-PI3K (\#4228), and anti-GAPDH (\#5174) antibodies were purchased from Cell Signaling Technology (USA). Goat anti-rabbit IgG antibody was purchased from Santa Cruz Biotechnology (USA).

\section{MTT assay}

Cells $\left(4 \times 10^{3}\right)$ were seeded in 96-well plate and treated with HYJD at various concentrations for the different times. After treatment, cells were incubated with $5 \mathrm{mg} /$ mL MTT $(10 \mu \mathrm{L} /$ well $)$ for 4 hours at $37^{\circ} \mathrm{C}$. MTT formazan production was dissolved by DMSO (200 $\mu \mathrm{L} /$ well). The plates were measured under a microplate Reader (BioTek, USA) at $570 \mathrm{~nm}$.

\section{Cell adhesion assay}

In brief, 96-well flat-bottom plates were coated with 5 $\mathrm{mg} / \mathrm{mL}$ matrigel $(200 \mu \mathrm{L} /$ well $)$ overnight at $4^{\circ} \mathrm{C}$. Cells $\left(5 \times 10^{4}\right)$ were treated with various concentrations of HYJD for 48 hours, then added into each matrigelcoated well in triplicate. After incubation for 3 hours, plates were washed three times with PBS to remove unbound cells. The bound cell was incubated with 5 $\mathrm{mg} / \mathrm{mL}$ MTT $(10 \mu \mathrm{L} /$ well $)$ for 4 hours at $37^{\circ} \mathrm{C}$. MTT formazan production was dissolved by DMSO $(200 \mu \mathrm{L} /$ well). The plates were measured under a microplate Reader (BioTek, USA) at $570 \mathrm{~nm}$. The percentage of bound cells was calculated by dividing the absorbance of the initial input cells.

\section{Transwell migration assay}

The transwell migration assay was determined using Transwell Cell Culture System (BD Biosciences, USA) according to the manufacturer's instructions. Briefly, the lower surface of the upper chambers was coated with fibronectin $(10 \mu \mathrm{g} / \mathrm{mL})$ at $37^{\circ} \mathrm{C}$ for 2 hours. Cells $(5$ $\times 10^{4}$ ) were treated with various concentrations of HYJD for 48 hours, then added into the upper chamber in serum-free medium. The bottom chamber contained medium with $20 \%$ FBS. After 24 hours, cells that had migrated through the membrane to the lower surface were evaluated by crystal violet staining. The number of migratory cells in 5 microscopic fields was counted.

\section{Cell invasion assay}

The matrigel invasion assay was determined using Transwell Cell Culture System (BD Biosciences, USA) according to the manufacturer's instructions. Briefly, the upper chambers were coated with $5 \mathrm{mg} / \mathrm{mL}$ matrigel $(100 \mu \mathrm{L} /$ well $)$ at $37^{\circ} \mathrm{C}$ for 4 hours. Cells $(5 \times$ $10^{4}$ ) were treated with various concentrations of HYJD for 48 hours, then added into the upper chamber in serum-free medium. The bottom chamber contained medium with $20 \%$ FBS. After 24 hours, cells that had invaded through the membrane to the lower surface were evaluated by crystal violet staining. The number of invasive cells in 5 microscopic fields was counted.

\section{Quantitative real-time polymerase chain reaction (qPCR)}

Cells were collected and total RNA was isolated using trizol reagent (Invitrogen, USA) according to the manufacturer's instructions. 2 ug RNA was used for reverse transcription with Oligo (dT) primers and reverse transcriptase (TaKaRa Biotechnology, Japan). Quantitative real-time PCR was performed with the ABI Prism 7000 sequence detection system (Applied Biosystems, Foster City, CA) using TaqMan PCR Master Mix and probes (Invitrogen, Carlsbad, CA). GAPDH was performed as a loading control.

\section{Western blot}

Briefly, tumor tissues were homogenized in RIPA 
buffer containing protease and phosphatase inhibitor cocktails (Cell Signaling Technology, USA). After centrifugation, the protein concentrations were determined using a BCA ${ }^{\mathrm{TM}}$ protein assay Kit (Pierce, USA). Equal amounts of total protein were mixed with SDS sample buffer, and separated by SDS-polyacrylamide gel electrophoresis. After electrophoresis, protein was electro-transferred onto a polyvinylidene diuoride membrane (Millipore Corp., USA) and blocked for 1 hour in 5\% nonfat milk. The membrane was incubated with primary antibodies at $4^{\circ} \mathrm{C}$ overnight and horseradish peroxidase (HRP)-conjugated secondary antibody for 1 hour at room temperature. Protein bands were visualized using enhanced chemiluminescence reagents according to the manufacturer's instructions (Thermo Scientific, USA). GAPDH was performed as a loading control. The densities of protein bands were determined by Image J software.

\section{PDK1 kinase activity assay}

PDK1 kinase activity was determined by HTScan ${ }^{\circledR}$ PKD1 kinase assay kit (Cell Signaling Technology, MA) in accordance to the manufacture's protocols. Briefly, PDK1 kinase was incubated with various concentrations of HYJD in reaction buffer at room temperature. After incubation for $30 \mathrm{~min}$, the buffer was transferred to streptavidin-coated plate and incubate for 1 hour. After washing three times with washing buffer, diluted phosphor-PKA/C Thr197 antibody was added and incubated for 2 hours. after washing three times, kinase activity was determined by colorimetric ELISA methods. The plates were measured under a microplate Reader (BioTek, USA) at $450 \mathrm{~nm}$.

\section{Tumor xenograft mouse model}

Male Balb/c athymic nude mice, 6-8 weeks of age, were obtained from Henan Academy Institute of Traditional Chinese Medicine (Henan, China). They were maintained with free access to pellet food and water in sterile filter-topped cages at a temperature of $21 \pm 2{ }^{\circ} \mathrm{C}$ with a 12 hours light/dark cycle. SGC-7901 cells $(1 \times$ $\left.10^{6}\right)$ were subcutaneously injected into the left parts of the mice. After injection for four days, mice formed tumor and then distributed into four groups according to tumor size measured by a caliper. Mice were administered with various concentrations of HYJD via gavage daily for two weeks. CTX was administered intraperitoneally $(30 \mathrm{mg} / \mathrm{kg} /$ day) daily as a positive control group. Negative control animals were injected with water. After two weeks administration, mice were sacrificed and tumor tissues were collected for analysis.

\section{Statistical analysis}

Statistical significance was determined using Student's t -tests and one-way analysis of variance (ANOVA) with SPSS18.0 software (SPSS Inc., Chicago, IL, USA). $\mathrm{p}<0.05$ was considered statistically significant.

\section{Results}

\section{HPLC analysis of extract and chemical profile}

As shown in Figure 1, HYJD was composed by a large number of single components. Peak 1 and peak 2 could be inferred as scutellarein and apigenin based on the chromatogram of standard product (Figure 1D). In addition, by the comparison of HPLC chromatogram, there was no obvious change among the peak area of the sample with different storage time (Figure 1A, B, C). Therefore, the extract of HYJD was stable.

\section{Proliferation of various cancer cell lines}

Various cancer cell lines including SGC-7901, A549, HepG2, Hela, Caco-2, MCF-7 and ECA-109 were incubated with different concentrations of HYJD for 48 hours. $50 \%$ inhibitory concentration ( $\mathrm{IC}_{50}$ values) were determined by MTT assay. As shown in Figure 2A, HYJD exhibited strong inhibitory effect on cell proliferation. $\mathrm{IC}_{50}$ values were around 1.23-6.23 mg/ mL. SGC-7901 cells were the most sensitive to HYJD treatment and used in further study. Moreover, HYJD treatment inhibited SGC-7901 cells proliferation in a dose- and time-dependant manner (Figure 2B and C).

\section{SGC-7901 cells adhesion, invasion and migration}

It is well known that adhesive, migratory and invasive abilities play crucial roles in metastasis of malignant tumor (Hood and Cheresh, 2002). We further investigated the effects of HYJD on these abilities of SGC-7901 cells. As shown in Figure 3A, after incubation for 3 hours, $42 \%$ cells were bound to the flat-bottom of plates. However, HYJD-treated cells (1.25 and $2.5 \mathrm{mg} /$ $\mathrm{mL}$ ) showed much weaker adhesive ability. Only 4-18\% cells were bound to the bottom of wells. Then we analyzed the migratory and invasive abilities of HYJDtreated cells. HYJD treatment showed a significant inhibition on migratory and invasive abilities of SGC7901 cells. As a positive control, CTX $(30 \mu \mathrm{M})$ also exhibited a strong inhibition on adhesive, migratoryand invasive abilities of SGC-7901 cells.

\section{Down-regulated phosphorylation of Akt protein in SGC-7901 cells}

Inhibition of AKT activity leads to aberration of proliferation and growth of tumor cells (Serra et al, 2008). In the following study, we determined the effects of HYJD on AKT pathway. Firstly, the effects of HYJD on AKT mRNA expression were determined by qPCR. As shown in Figure 4A, HYJD had no effect on PI3K, PDK1 and AKT mRNA expression in SGC-7901 cells. However, western blot results showed that HYJD treatment markedly down-regulated phosphorylation of AKT protein level with no effect on PI3K and PDK1 in SGC-7901 cells. CTX $(30 \mu \mathrm{M})$ also significantly downregulated phosphorylation of AKT protein level. 


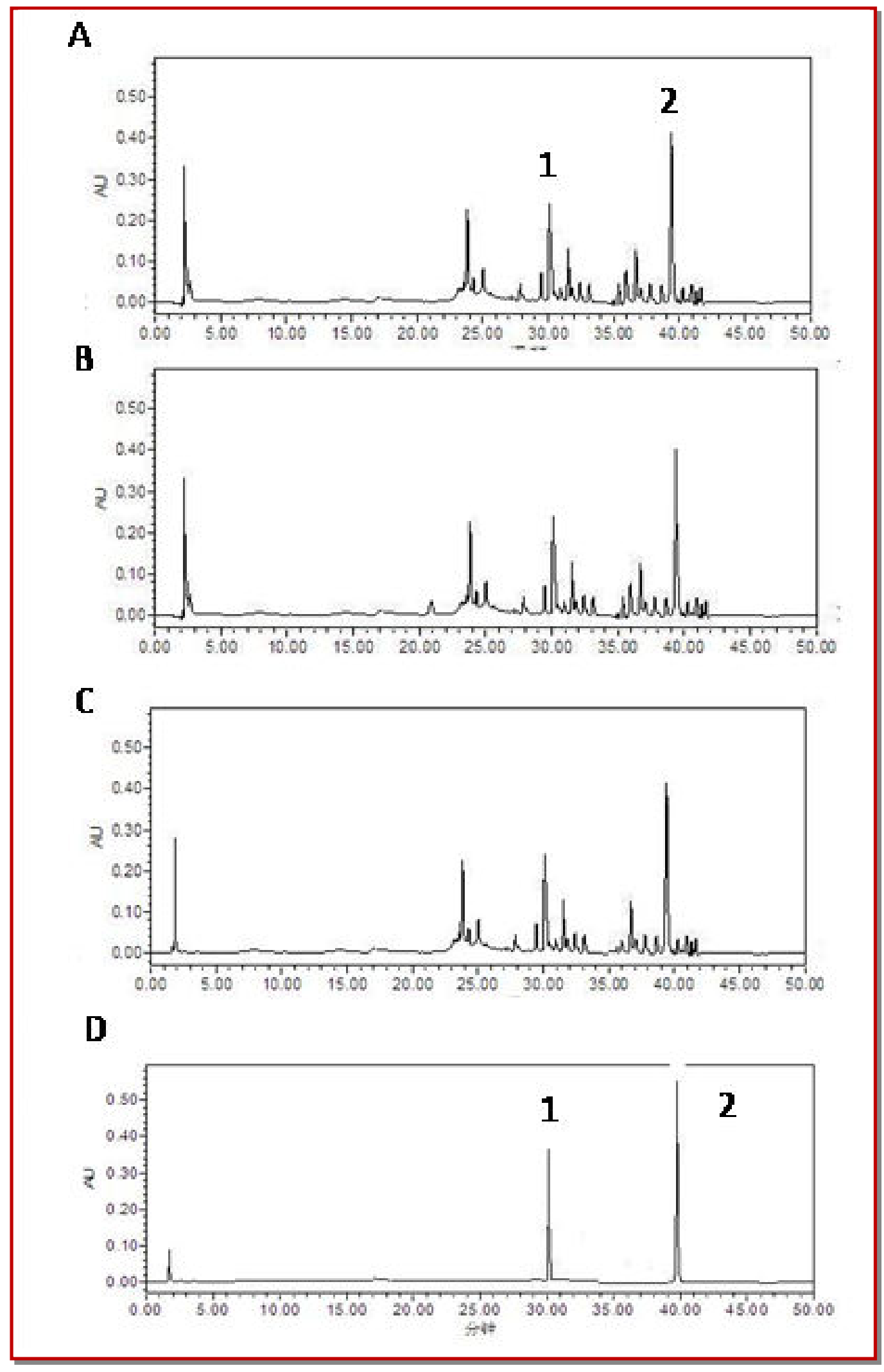

Figure 1: HPLC analysis of extract of HYJD and chemical profile. (A): HPLC chromatogram of HYJDE stayed for one day; B: HPLC chromatogram of HYJDE stayed for 3 months; C: HPLC chromatogram of HYJDE stayed for 9 months; D: HPLC chromatogram of the standard sample. Peak 1: Scutellarein; Peak 2: Apigenin 

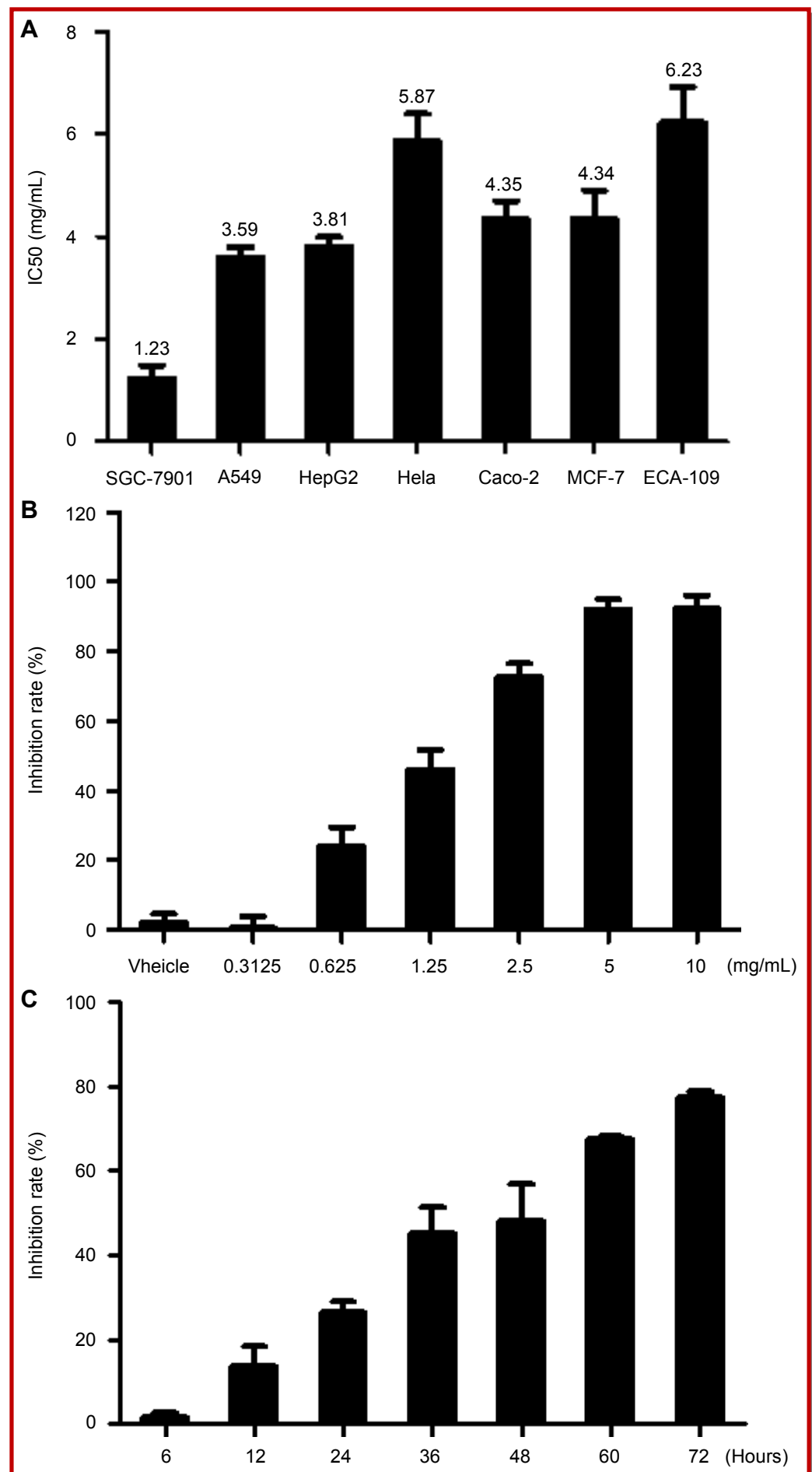

Figure 2: HYJD inhibited proliferation of various cancer cell lines. (A) Various cancer cell lines including SGC-7901, A549, HepG2, Hela, Caco-2, Hela, MCF-7 and ECA-109 were treated with different concentrations of HYJD for 48 hours. Cell viability were determined by MTT assay and 50\% inhibitory concentration (IC50 values) were calculated. (B) SGC-7901 cells were treated with indicated concentration of HYJD for 48 hours. (C) SGC-7901 cells were treated with $1.25 \mathrm{mg} / \mathrm{mL}$ HYJD for the different times. Cell viability were determined by MTT assay. Data are shown as means \pm S.E.M of three independent experiments 


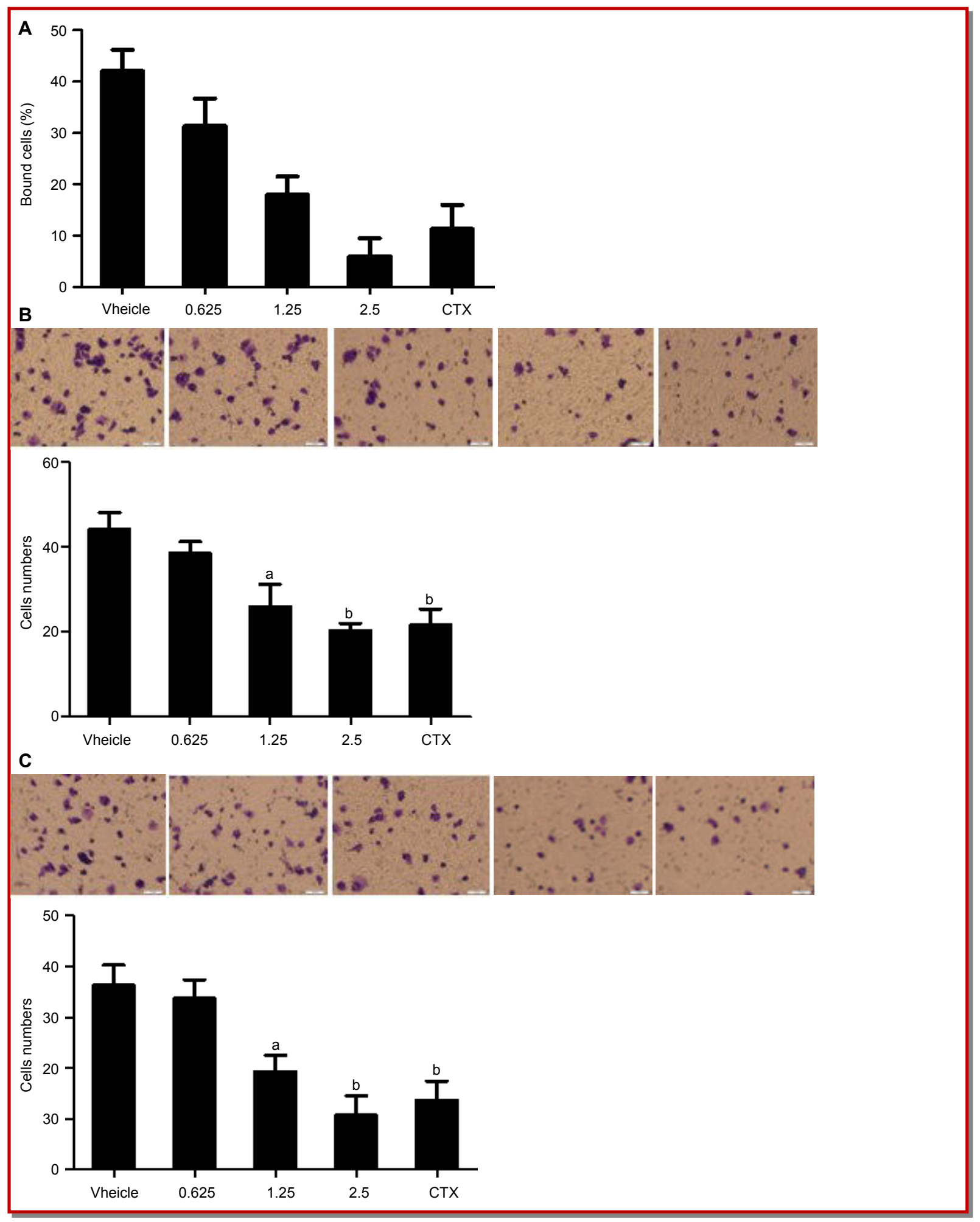

Figure 3: HYJD significantly inhibited adhesive, migratoryand invasive abilities of SGC-7901 cells. (A) Cells were treated with various concentrations of HYJD for 48 hours, then added into each Matrigel-coated well in triplicate. After incubation for 3 hours, the bound cells were analyzed by MTT assay. (B) The lower surface of the upper chambers was coated with $10 \mu \mathrm{g} / \mathrm{mL}$ fibronectin. (C) The upper chambers were coated with $5 \mathrm{mg} / \mathrm{mL}$ Matrigel. Cells were treated with various concentrations of HYJD for 48 hours, then added into the upper chamber of transwell in serum-free medium. The bottom chamber contained medium with $20 \%$ FBS. After 24 hours, cells that had migrated or invaded through the membrane to the lower surface were evaluated by crystal violet staining. The numbers of cell in 5 microscopic fields was counted. Data are shown as means \pm S.E.M of three independent experiments. a $\mathrm{P}<0.05, \mathrm{~b}<<0.01$ 


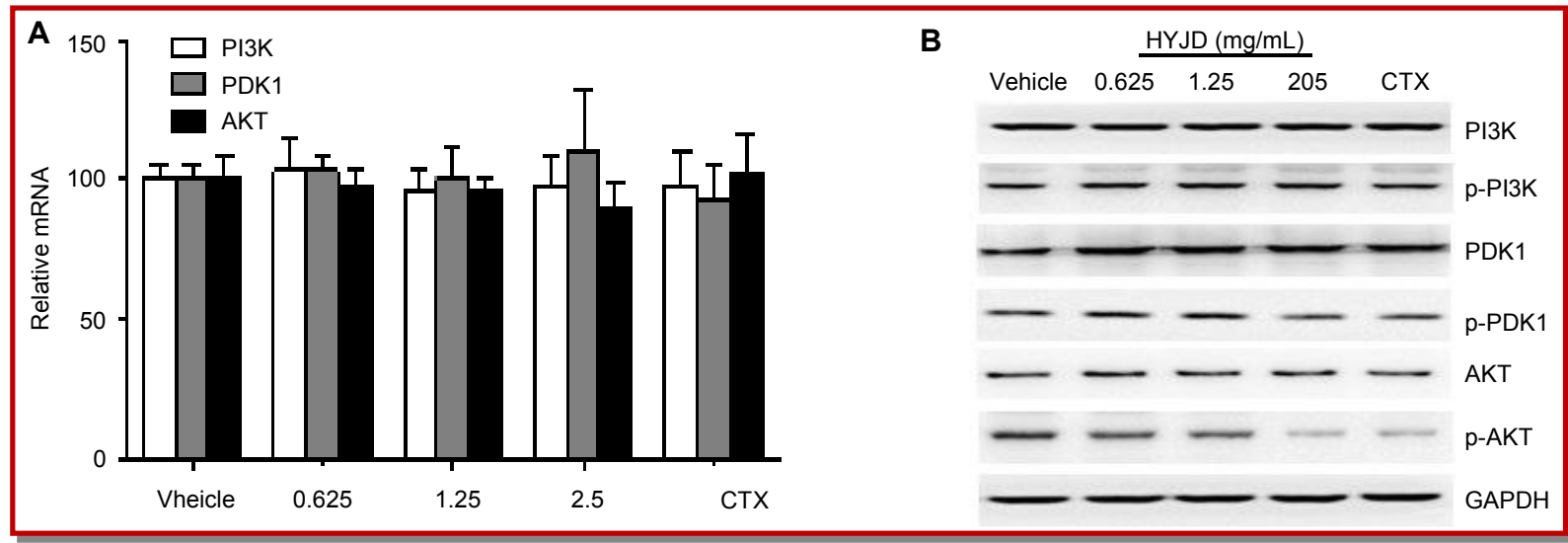

Figure 4 : HYJD down-regulated phosphorylation of Akt protein in SGC-7901 cells. (A) Cells were treated with HYJD at different concentrations or $30 \mu \mathrm{M}$ CTX for 12 hours. Total RNA from SGC-7901 cells was isolated and mRNA levels of PI3K, PDK1 and AKT were determined by qPCR. (B) Cells were treated with HYJD at different concentrations or $30 \mu \mathrm{M}$ CTX for 3 hours. The levels of PI3K, PDK1 and AKT protein were determined by western blot. Bands were analyzed by densitometry. GAPDH was used as the endogenous control. Data are shown as means \pm S.E.M of three independent experiments. ${ }^{a} p<0.05,{ }^{b} p<0.01$

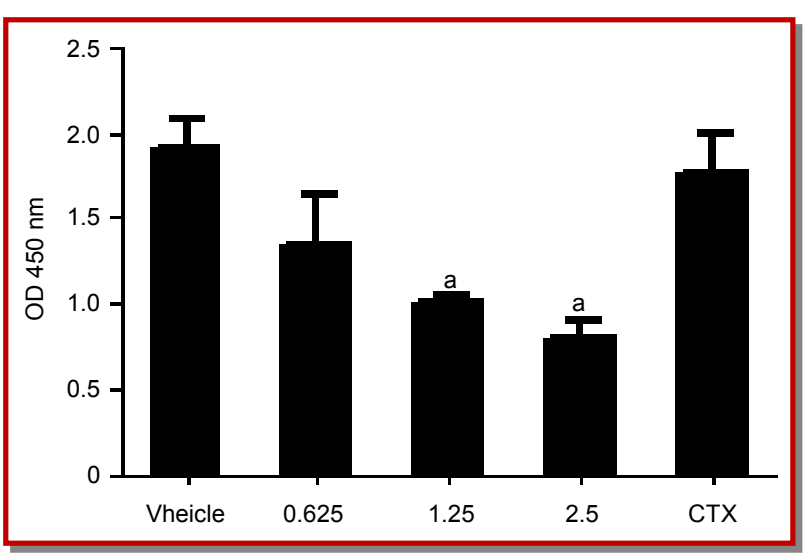

Figure 5: HYJD dramatically suppressed PDK1 kinase activity in vitro. PDK1 kinase activity was determined by HTScan ${ }^{\circledR}$ PKD1 kinase assay kit. Data are shown as means \pm S.E.M of three independent experiments. ${ }^{\mathrm{a}} \mathrm{p}<0.05,{ }^{\mathrm{b}} \mathrm{p}<0.01$

\section{PDK1 kinase activity in vitro}

As an upstream kinase of AKT protein, PDK1 phosphorylates and activates AKT (Shen et al, 2010). We further investigated whether down-regulated phosphorylation of Akt protein by HYJD treatment is due to inhibition of PDK1 kinase activity. PDK1 kinase (50 ng) was incubated with various concentrations of HYJD for $30 \mathrm{~min}$. As shown in Figure 5, HYJD treatment significantly inhibited PDK1 kinase activity in a dose dependent manner. As a positive control, CTX could not inhibited PDK1 kinase activity.

\section{Growth of tumor xenografts in vivo}

To investigate anti-tumor activity of HYJD in vivo, we injected SGC-7901 cells into the left parts of athymic nude mice. After all mice formed tumors, different doses of HYJD were administered via gavage daily for two weeks. As shown in Figure 6A and B, HYJD at the doses of $1 \mathrm{~g} / \mathrm{kg}$ and $2 \mathrm{~g} / \mathrm{kg}$ significantly inhibited weights of tumor xenografts. $30 \mathrm{mg} / \mathrm{kg}$ CTX, as a positive control, also significantly inhibited tumor weight. Moreover, HYJD administration has little impact on body weight of mice and the spleen index, whereas CTX decreased body weights and the spleen index of nude mice than control group (Figure 7).

\section{Phosphorylation of Akt protein in tumor tissue}

Next, we determined phosphorylation of AKT proteins in tumor xenografts using Western blot. Consistent with result in vitro, western blot data showed that HYJD significantly decreased phosphorylation of AKT protein level in tumor tissue (Figure 8).

\section{Discussion}

In this study, we investigated the effects of HYJD, a TCM formula, on anti-tumor activity in vitro and in vivo. We screened various tumor cell lines, and found that HYJD exhibited the strongest cytotoxicity to gastric cancer cell line SGC-7901 compared to other cell lines. It is well known that traditional Chinese medicine has held, and still holds, an important position in primary health care in China because of its 5000 year old tradition (Zhang et al, 2013). Natural products from TCM represent a valuable source for the identification of small-molecule inhibitors against novel targets for medicine, especially for cancer therapy (Efferth et al, 2007). Therefore, to explore new intervention of TCM is critical and urgent for cancer prevention and treatment.

Numerous studies have been reported that approximately $90 \%$ of cancer deaths were attributable to the presence of distant metastasis (Hanahan and Weinberg, 2000). Associated with many molecules, metastasis includes cell adhesion to extracellular matrix, migration towards specific direction and 


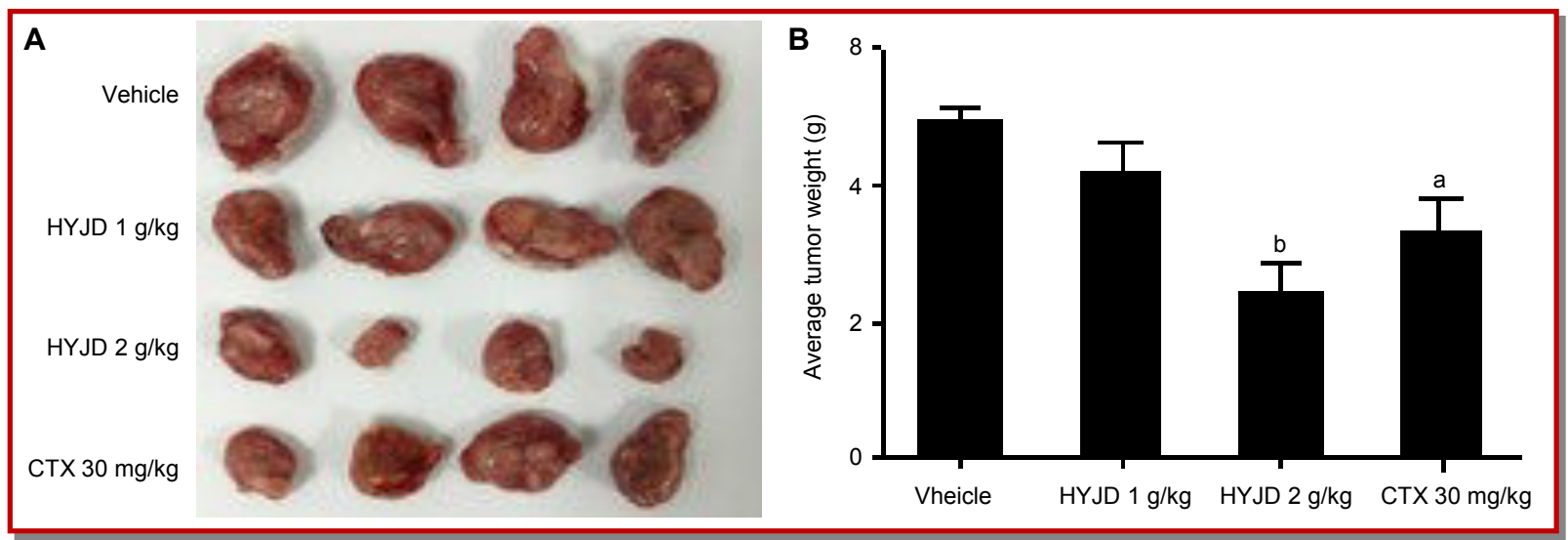

Figure 6: HYJD inhibited the growth of tumor xenografts in vivo. SGC-7901 cells were subcutaneously injected into the left part of the mice. Indicated doses of HYJD were administered via gavage daily for two weeks. CTX $(30 \mathrm{mg} / \mathrm{kg})$ was administered intraperitoneally as a positive control group. After two weeks, mice were sacrificed and SGC-7901 xenograft tumors were photographed and analyzed. Data are shown as means \pm S.E.M. ${ }^{a} \mathrm{p}<0.05,{ }^{\mathrm{b}} \mathrm{p}<0.01$

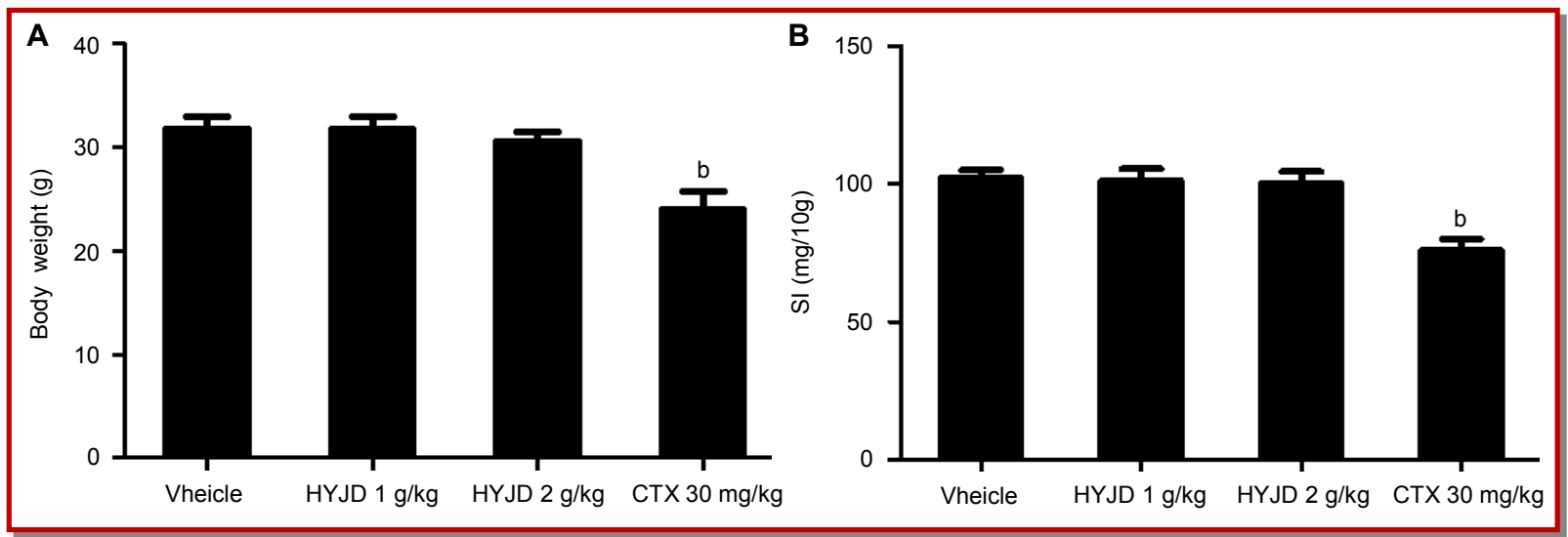

Figure 7: The effects of HYJD on body weight and spleen index. Mice treated with HYJD were sacrificed and body weight and the spleen index (SI, spleen/body weight) were measured. Data are shown as means \pm S.E.M. ${ }^{a} p<0.05,{ }^{b} p<0.01$

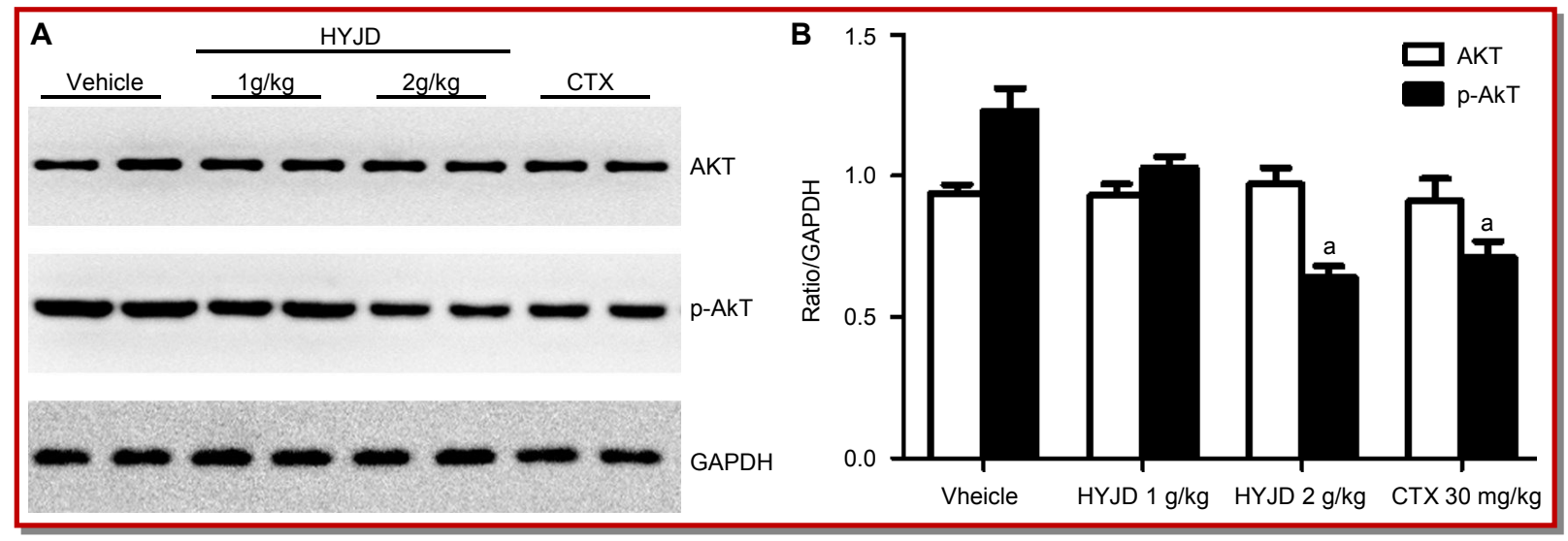

Figure 8: HYJD down-regulated phosphorylation of Akt protein in tumor tissue. The phosphorylation of AKT protein level in tumor tissue was determined by western blot. Bands were analyzed by densitometry. GAPDH was used as the endogenous control. Data are shown as means \pm S.E.M. ${ }^{a} p<0.05,{ }^{b} p<0.01$ 
invasion into local vessel of distant organs (Wang et al, 2008). Our results revealed that HYJD treatment markedly inhibited adhesion, migration and invasion abilities of SGC-7901 cells. The increasing evidences have suggested that the huge number of active ingredients in one formula makes this formula suitable for multi-target actions (Jiang, 2005). As a TCM formula, HYJD consists of six Chinese medicines and exhibits multiple targets and lower toxicity, which contributed to multiple steps of tumor metastasis such as adhesion, migration and invasion. Therefore, multicomponent therapeutics offer bright prospects for the control of complex diseases.

Recently, the number of molecular targets used for developing novel treatment options increased considerably. Among these targets are many signaltransduction proteins (e.g. kinases) that regulate malignant processes in cancer cells (Hidalgo and Rowinsky, 2000). AKT is a threonine/serines protein kinase, and has been reported to be a key regulator in survival growth of tumors (Schmidt et al, 2002). It was found that HYJD dose-dependently down-regulated phosphorylation of AKT protein levels in vitro and in vivo. According to abundant reports mentioned, many protein as the upstream signal protein phosphorylated AKT kinase. In order to investigate how HYJD inhibited phosphorylation of AKT protein, we screened various related kinases. Interestingly, we found that PDK1 kinase activity, an upstream protein of AKT, was dramatically inhibited by HYJD in a dose-dependent manner. Phosphoinositide 3-kinases (PI3K) are a family of related intracellular signal transducer enzymes involved in cellular functions such as cell growth, proliferation, differentiation, motility, survival and intracellular trafficking, which in turn are involved in cancer (Engelman, 2009). As a downstream protein of PI3K, Phosphoinositide-dependent protein kinase 1 (PDK1) plays a central role in many signal transduction pathways including the activation of Akt and the PKC isoenzymes p70 S6 kinase and RSK (Belham et al, 1999; Toker and Newton, 2000). Our data suggested that HYJD might directly target PDK1 and subsequently inhibited phosphorylation of AKT protein. However, CTX could not inhibit PDK1 kinase activity, suggesting that CTX may inhibit other upstream protein of Atk to decrease Akt phosphorylation. Like abundant kinases, telomerase as a drug target plays an important role in the immortalization of tumor cells (Sampedro Camarena et al, 2007; Zimmermann and Martens, 2007). It has been reported that small-molecule inhibitors derived from Chinese medicinal plants exhibited inhibition of telomerase activity (Zhang et al, 2002).

\section{Conclusion}

Extracts of HYJD exhibited an anti-tumor activity both in vitro and in vivo. HYJD treatment inhibited cell proliferation, adhesion, migration and invasion abilities. Moreover, HYJD down-regulated phosphorylation of AKT protein level through blocking the activity of PDK1 kinase. Our data suggested that HYJD may be a potential drug in the treatment of cancer.

\section{Ethical Issue}

All animal experiments were approved by the Ethics Committee for Animal Experimentation, Henan Academy institute of Traditional Chinese Medicine.

\section{Acknowledgement}

Authors acknowledge the support of National Natural Science Foundation of China (Grand No.81273877and 81403267).

\section{References}

Belham C, Wu S, Avruch J. Intracellular signalling: PDK1--a kinase at the hub of things. Curr Biol. 1999; 9: R93-96.

Cao Y, Xia QH, Meng H, Zhong AP. Anti-tumor and synergistic effect of Chinese medicine "bushen huayu jiedu recipe" and chemotherapy on transplanted animal hepatocarcinoma. World J Gastroenterol. 2005; 11: 5218-20.

Cao Y, Xia QH, Meng H, Zhong AP. Pharmacological effects of serum containing Chinese medicine Bushen Huayu Jiedu compound recipe in lung cancer drug-resistance cells. Chinese J Integr Med. 2008; 14: 46-50.

Efferth T, Li PC, Konkimalla VS, Kaina B. From traditional Chinese medicine to rational cancer therapy. Trends Mol Med. 2007; 13: 353-61.

Engelman JA. Targeting PI3K signalling in cancer: Opportunities, challenges and limitations. Nat Rev Cancer. 2009; 9: $550-62$.

Hanahan D, Weinberg RA. The hallmarks of cancer. Cell 2000; 100: $57-70$

Hidalgo M, Rowinsky EK. The rapamycin-sensitive signal transduction pathway as a target for cancer therapy. Oncogene 2000; 19: 6680-86.

Hood JD, Cheresh DA. Role of integrins in cell invasion and migration. Nat Rev Cancer. 2002; 2: 91-100.

Jemal A, Bray F, Center MM, Ferlay J, Ward E, Forman D. Global cancer statistics. CA Cancer J Clin. 2011; 61: 69-90.

Jiang WY. Therapeutic wisdom in Traditional Chinese Medicine: A perspective from modern science. Discovery Med. 2005; 5: 455-61.

Li X, Yang G, Li X, Zhang Y, Yang J, Chang J, Sun X, Zhou X, Guo Y, Xu Y, Liu J, Bensoussan A. Traditional Chinese Medicine in cancer care: A review of controlled clinical studies published in Chinese. PLoS One 2013; 8: e60338.

Sampedro Camarena F, Cano Serral G, Sampedro Santalo F. 
Telomerase and telomere dynamics in ageing and cancer: Current status and future directions. Clinical and translational oncology: Official publication of the Federation Spanish Oncology Societies and of the National Cancer Institute of Mexico. 2007; 9: 145-54.

Schmidt M, Hovelmann S, Beckers TL. A novel form of constitutively active farnesylated Akt1 prevents mammary epithelial cells from anoikis and suppresses chemotherapyinduced apoptosis. Bri J Cancer. 2002; 87: 924-32.

Serra V, Markman B, Scaltriti M, Eichhorn PJ, Valero V, Guzman M, Botero ML, Llonch E, Atzori F, Di Cosimo S, Maira M, Garcia-Echeverria C, Parra JL, Arribas J, Baselga J. NVP-BEZ235, a dual PI3K/mTOR inhibitor, prevents PI3K signaling and inhibits the growth of cancer cells with activating PI3K mutations. Cancer Res. 2008; 68: 8022-30.

Shen X, Xi G, Radhakrishnan Y, Clemmons DR. PDK1 recruitment to the SHPS-1 signaling complex enhances insulin-like growth factor-i-stimulated AKT activation and vascular smooth muscle cell survival. J Biol Chem. 2010; 285: 2941624.
Siegel R, Ma J, Zou Z, Jemal A. Cancer statistics, 2014. CA Cancer J Clin. 2014; 64: 9-29.

Toker A, Newton AC. Cellular signaling: Pivoting around PDK -1. Cell 2000; 103: 185-88.

Wang L, Zhou X, Zhou T, Ma D, Chen S, Zhi X, Yin L, Shao Z, Ou Z, Zhou P. Ecto-5'-nucleotidase promotes invasion, migration and adhesion of human breast cancer cells. J Cancer Res Clin Oncol. 2008; 134: 365-72.

Zhang F, Jia Z, Deng Z, Wei Y, Zheng R, Yu L. In vitro modulation of telomerase activity, telomere length and cell cycle in MKN45 cells by verbascoside. Planta Medica. 2002; 68: 115-18.

Zhang L, Zhang J, Chen J, Xing D, Mu W, Wang J, Shang H. Clinical research of Traditional Chinese Medicine needs to develop its own system of core outcome sets. Evid Based Complement Alternat Med. 2013; 2013.

Zimmermann S, Martens UM. Telomeres and telomerase as targets for cancer therapy. Cell Mol Life Sci. 2007; 64: 906-21. 


\section{Your feedback about this paper}

1. Number of times you have read this paper 0

2. Quality of paper
Excellent
Good
Moderate
Not good

3. Your comments 